\title{
Criminologie
}

\section{La sociolinguistique au secours des juristes}

\section{Claude Tousignant}

Volume 24, numéro 1, 1991

Les droits des détenu-e-s

URI : https://id.erudit.org/iderudit/017305ar

DOI : https://doi.org/10.7202/017305ar

Aller au sommaire du numéro

Éditeur(s)

Les Presses de l'Université de Montréal

ISSN

0316-0041 (imprimé)

1492-1367 (numérique)

Découvrir la revue

Citer cet article

Tousignant, C. (1991). La sociolinguistique au secours des juristes. Criminologie, 24(1), 105-120. https://doi.org/10.7202/017305ar

\section{Résumé de l'article}

Over the past twenty or so years, more and more linguists have been called upon to give evidence in U.S. and Canadian Courts. The fields in which they have provided testimony include that of socidinguistic analysis based on the link-up between language and society. The evidence they provide of the testimony between the socio-economic background of an accused person and his or her linguistic production may, for example, determine the validity of a confession or the justification for a libellous statement attributed to the accused. However, the lawyer who is familiar with and can master the various social connotations associated with the various expressions used in court will have a major advantage when it comes to encouraging a witness to speak out, or convincing the judge and jury of the credibility of what he or she tells them. 
Over the past twenty or so years, more and more linguists have been called upon to give evidence in U.S. and Canadian Courts. The fields in which they have provided testimony include that of sociolinguistic analysis based on the link-up between language and society. The evidence they provide of the testimony between the socio-economic background of an accused person and his or her linguistic production may, for example, determine the validity of a confession or the justification for a libellous statement attributed to the accused. However, the lawyer who is familiar with and can master the various social connotations associated with the various expressions used in court will have a major advantage when it comes to encouraging a witness to speak out, or convincing the judge and jury of the credibility of what he or she tells them.

La sociolinguistique est la science qui établit le corrélat existant entre la structure sociale et la structure linguistique. Elle s'inspire donc du statut social du locuteur ainsi que de la situation dans laquelle il évolue et rattache à ces paramètres un modèle linguistique adéquat.

Les recherches sociolinguistiques ont pu établir plusieurs postulats par le biais de centaines d'études réalisées partout dans le monde à partir de «corpus» (ou données d'analyse recueillies par le chercheur). Ceux-ci ont surtout trait à la langue parlée mais peuvent également provenir de documents écrits (tels articles de journaux, annonces publicitaires, etc.). Parmi les conclusions les plus importantes auxquelles ont mené ces recherches, on peut dégager celles qui suivent.

\subsection{LANGAGE ET STATUT SOCIAL}

Il existe un lien formel entre le statut social du sujet et sa façon d'utiliser la langue parlée ou écrite. L'on entend par statut social tout état de fait caractérisant l'individu à l'intérieur d'une société. Ainsi, des paramètres tels le degré de scolarité, le type d'emploi, le niveau socio-économique du quartier, etc., sont autant de facteurs qui se situent à l'origine de ce que nous appelons

* Professeur de linguistique au Département de français, Université du Québec à Trois-Rivières; chercheur associê au Centre d'études québécoises, Case postale 500, TroisRivières (Québec) G9A 5H7. 
les classes sociales et constituent du même fait des indicateurs et des balises du type de langage prôné par l'humain. Ces facteurs présentent souvent un lien très étroit avec certaines tendances et certaines habitudes linguistiques.

Les sociolinguistes ont maintes fois mis en lumière les relations tantôt entre le fait d'être scolarisé et celui d'adopter telle habitude discursive, tantôt entre le fait d'occuper un emploi de type manuel et celui d'utiliser telle autre forme langagière. Ce lien se résume souvent de la façon suivante : un locuteur entretenant des contacts réguliers avec la norme (celle des grammaires et dictionnaires) aura tendance à recourir à des procédés similaires dans l'utilisation de sa propre langue écrite et même de sa langue parlée. Ainsi, les sujets possédant une profession leur procurant ce contact régulier avec l'écrit (professeurs, avocats, écrivains, journalistes, professions libérales en général...) figureront habituellement parmi ceux dont la langue sera la plus standard, la plus normative. Dans le même ordre d'idées, l'on peut dire que plus le degré de scolarité d'un locuteur est élevé, plus celui-ci sera enclin à employer une langue normative, châtiée ${ }^{1}$.

En contrepartie, nous pouvons également dire que moins un sujet est scolarisé (donc moins il a été mis en contact avec cette norme), plus il sera appelé à utiliser des formes présentant un écart important par rapport à la langue écrite standard. C'est là que nous retrouverons par exemple des formes syntaxiques non compatibles avec la norme telles que «la fille que je sors avec!», ou l'emploi de termes appartenant exclusivement à la langue parlée populaire (ex.: «se fendre en quatre», «être tanné», etc.).

\subsection{LANGAGE ET CONTEXTE SOCIAL}

La situation dans laquelle se déroule l'entretien jouera également un rôle de premier ordre dans la nature des composantes linguistiques. Ainsi, une situation de grande formalité (telle une demande d'emploi quant à la langue écrite ou une conférence devant un public adulte quant à la langue parlée) sera caractérisée par l'utilisation de formes normatives plutôt que populaires. Celles-ci seront fonction du degré d'attention porté par le sujet à sa langue (ou «autocorrection»), lequel sera lui-même à l'origine de ce que l'on appelle les «niveaux de langue» (différents toutefois selon qu'ils appartiennent à la

1. En fait, ce principe est celui du miroir puisque les instances politico-sociales ayant le pouvoir de juger qu'une forme est normative ou non, donc que cette forme peut ou non constituer un élément de l'écrit (alors manipulé par l'apprenant pendant sa période scolaire), sont les mêmes qui jugeront du langage de l'individu. On pourrait ajouter que le sujet qui, en contrepartie, manipule ces formes avec brio pourrait éventuellernent être appelé à faire partie de cette élite politico-sociale. 
langue parlée ou écrite ${ }^{2}$ ). Par contre, une conversation intime entre deux amis ou une lettre adressée à un parent feront plutôt appel à un code plus spontané, plus à l'abri des contraintes prescriptives de la norme. Cela signifie donc qu'un homme pourra utiliser une forme $x$ pendant le petit déjeuner avec son épouse mais opter pour une forme $y$ quand il donnera son cours au cégep ou s'il témoigne devant un juge. De la même façon, cet homme pourra écrire à son épouse dans un style $x$ et à son député dans un style $y$, avec, dans ce dernier cas, un vocabulaire plus recherché. C'est ce que l'on appelle la dimension psychologique du discours.

\subsection{LANGAGE ET GÉOGRAPHIE}

Une troisième cause pouvant influer sur la nature exacte du langage a trait à l'aspect géographique entourant la production linguistique. Celle-ci sera souvent porteuse de formes lexicales ou phonétiques particulières. Ainsi, le sujet originaire de Trois-Rivières emploiera le terme «chétif» (prononcé chéti) en faisant allusion à un personnage désagréable pendant que le sujet montréalais l'associera plutôt à une personne maigre, rachitique (en prononçant toutefois le $f$ final). Cette distinction au niveau du sens et de la prononciation est imputable au fait que les utilisateurs de ces formes, habitant des régions différentes, seront moins appelés à entrer en interaction linguistique et adopteront alors des usages qui nous laisseront entrevoir des différences parfois manifestes. Nous parlerons alors de «dialectes» différents.

L'absence de contacts suivis (autant du point de vue de leur fréquence que de leur intimité), causée dans le cas précité par une distance physique, est parfois attribuable à plus d'un facteur. Ainsi la présence d'une ville ou d'un village entre deux agglomérations (même relativement rapprochées l'une de l'autre) sera parfois suffisante pour engendrer des différences de prononciation discernables par l'ensemble des sujets. Même des accidents terrestres telles les chaînes de montagnes ou les grandes étendues d'eau seront susceptibles d' «isoler» en quelque sorte les habitants d'une région et d'entraîner par là une disparité linguistique non négligeable.

Sans vouloir toutefois minimiser l'impact de l'aspect géographique sur la variabilité du langage, nous nous devons tout de même d'en restreindre quelque peu la portée. L'importance et l'omniprésence caracterisant en effet

2. On a en effet tendance à classer différemment les niveaux de langue selon qu'ils appartiennent à l'oral ou à l'écrit. Ainsi un niveau dit «correct» en langue parlée sera perçu comme étant familier en langue écrite alors qu'un niveau dit «soutenu» en langue parlée apparaîtra à peine correct pour l'écrit. Finalement une langue parlée dite "rrecherchée» se verra attribuer le qualificatif «littéraire» si elle est transposée à l'écrit. La seule exception réside dans le niveau populaire qui, invariablement, s'appliquera à l'oral comme à l'écrit. 
les réseaux de communication et de diffusion depuis quelques années nous obligent à reconnaître un rôle beaucoup plus limité à cette distance géographique à l'égard des distinctions linguistiques. La pénétration qu'exercent les médias écrits et électroniques dans les régions les plus éloignées contribue à une certaine homogénéité des formes langagières caractérisant les divers groupements d'individus. De façon générale, l'on a de plus en plus tendance à «imiter» les habitudes discursives des grands centres urbains, ceux-là mêmes qui possèdent habituellement le monopole des réseaux de diffusion.

Ainsi, lorsque Radio-Canada, par exemple, diffuse son téléjournal dans les régions périphériques du Québec et du reste du Canada, il est évident que le lecteur de nouvelles et les correspondants risquent, à longue échéance, d'influencer leurs auditeurs assidus. La plupart de ces émissions étant produites à Montréal, c'est donc l'accent montréalais («rehaussé» d'une prononciation très standard) qui se fera entendre dans des régions aussi nombreuses que variées ${ }^{3}$.

\subsection{LANGAGE ET ÂGE}

L'âge du locuteur pourra également influer sur son expression linguistique. Par exemple, plusieurs enquêtes ont démontré que les jeunes locuteurs sont habituellement facteurs d'innovation linguistique (production de nouvelles formes dans la langue parlée ou écrite), étant beaucoup plus à l'affût de ce qui crée un effet de nouveauté dans le discours. Par ailleurs, les gens âgés (et scolarisés) auront souvent tendance à conserver des formes linguistiques ayant connu leur «heure de gloire» plusieurs années auparavant, ce que nous pourrions qualifier d'anciennes normes, lesquelles trahiront parfois l'âge des sujets les employant (Tousignant, 1987).

\subsection{LANGAGE ET CONNOTATION}

Comme nous venons de le voir très brièvement ${ }^{4}$, la variation linguistique relève de divers types de facteurs qui seront à même d'orienter fortement la probabilité d'apparition des formes discursives dans un sens ou dans l'autre. Cela signifie donc que si une formulation linguistique peut varier en fonction de tous ces critères, elle sera alors sujette à produire un effet qui sera variable lui aussi. On parlera alors de connotation d'une forme linguistique. De la sorte, la forme appartenant majoritairement à la classe populaire, à une situation

3. Ce phénomène observé au Québec s'applique également à la France où. par le biais des médias, la "parisianisation» (ou l'art de s'exprimer avec un accent parisien) a connu depuis un siècle un essor considérable, et ce aux dépens des dialectes régionaux.

4. Pour plus d'informations à ce sujet, voir Labov (1978), Marcellesi et Gardin (1974) et Garmadi (1981). 
familière, à une région éloignée des grands centres urbains possédera une connotation parfois négative dans certains milieux.

Par exemple, le mot «père» prononcé pére sera souvent perçu au Québec comme faisant partie du discours de la classe populaire. Sa connotation pourrait être négative dans les milieux intellectuels, scolarisés, etc. De la même façon, l'utilisation de termes recherchés, de mots savants, dans un contexte informel, d'intimité, pourrait entraîner certains jugements négatifs à l'égard de son utilisateur. Par exemple, un ouvrier québécois dans une taverne, sympathisant avec ses compagnons de travail et déclarant:

\section{[1] Vous avez vu ces congères 5 ?}

risquerait de subir quelques railleries et moqueries. Il va donc de soi que le phénomène de l'alternance linguistique est intimement lié au contexte social et n'est pas imputable au hasard. Cette constatation est d'une importance ultime dans la mesure ou elle permettra une certaine prédictibilité des formes en fonction du contexte.

\section{APPORT ET PERTINENCE DE LA SOCIOLINGUISTIQUE EN COUR DE JUSTICE}

Les procès, contrats, testaments deviennent généralement litigieux sur la simple base d'une phrase, d'un mot - ou même d'un son - mal défini, mal compris ou mal interprété. C'est ainsi que, souventes fois, un libelle ou une menace de mort ne reposent véritablement que sur un ou quelques mots, une phrase tout au plus. C'est la raison pour laquelle nous opterons, dans les pages qui suivent, pour une approche davantage phrastique que discursive, plus microscopique que macroscopique, nous attandant au substantif ou à l'épithète, à l'expression ou à l'énoncé. Cette approche, une parmi d'autres, n'implique pas pour autant que nous rejetions l'analyse plus globaliste du texte ou du discours en milieu juridique; au contraire, nous croyons que cette dernière s'avère d'une très grande pertinence dans la compréhension maximale du processus langagier judiciaire.

Les généralités résultant des recherches sociolinguistiques trouveront un champ d'application juridique sous plusieurs angles. En voici deux :

a) le statut social d'un prévenu et sa production linguistique;

b) la connotation sociale des formes linguistiques véhiculées en cour.

5. Communément appelées «bancs de neige» au Québec. 


\subsection{LE STATUT SOCIAL D'UN PRÉVENU ET SA PRODUCTION LINGUISTIQUE}

\subsubsection{Libelles et menaces de mort}

Il arrive fréquemment que le procès d'un prévenu s'articule autour de propos qui auraient été tenus à l'égard d'un autre individu. Tel pourrait être le cas, par exemple, lorsqu'il y a accusation de libelle. L'on prête alors à l'accusé certaines paroles qui, dans un contexte particulier, peuvent porter tout le poids des accusations qui pèsent sur les épaules de son pseudo-auteur. Souventes fois, dans de tels cas, aucune pièce justificative telle que document sonore ou audio-visuel n'accompagne la preuve de la poursuite. Seules les déclarations des témoins permettent de maintenir les accusations et leurs propos prennent alors une importance capitale dans le déroulement du procès.

C'est précisément à ce moment qu' il convient de se demander si les mots faisant partie des propos que l'on prête à l'accusé sont tous susceptibles d'appartenir au lexique actif ${ }^{6}$ de l'accusé. En d'autres mots, cela revient à poser le problème suivant:

\section{$N^{\prime}$ importe qui peut-il dire ou écrire n' importe quoi?}

La réponse est évidemment «non». Et les raisons peuvent en être aussi nombreuses que variées. Dans la plupart des cas, ce sont des facteurs d'ordre sociolinguistique qui nous permettront de mettre en doute la paternité de propos diffamatoires chez un prévenu. Tel que noté plus haut, l'on peut classer ces facteurs de la façon suivante:

a) le statut social du sujet;

b) le contexte social entourant l'échange linguistique;

c) l'origine géographique du sujet;

d) l'âge du sujet.

\section{A. Le statut social du sujet}

À titre d'illustration de facteurs liés au statut social, prenons le degré de scolarité d'un prévenu. Par exemple, il est possible qu'un mot ou une expression ne puisse faire partie du lexique d'un individu parce qu'une scolarité trop modeste ne lui aurait pas permis d'avoir accès à un vocabulaire si recherché. Tel serait le cas d'un prévenu dont la scolarité serait de cinq années et qui serait accusé d'avoir affublé son voisin des épithètes suivantes:

6. Mots pouvant non seulement être compris mais aussi produits par un sujet. 
[2] fumiste

vide-gousset

vilipendeur

tonitruant

mythomane

Et la liste pourrait être longue! Il est effectivement peu probable qu'un vocabulaire aussi «riche» (et aussi rare!) puisse appartenir au lexique d'un individu peu scolarisé. Nous ne prétendons toutefois pas qu'il soit impossible qu'un tel vocabulaire se retrouve sur les lèvres de cette personne, mais néanmoins nous croyons qu'une telle cause devient dès lors défendable (surtout si la pseudo-victime de ce libelle ou toute autre personne agissant à titre de témoin auditif dans cette affaire possède un degré de scolarité appréciable, lequel en ferait une personne susceptible de posséder ces éléments du discours dans son propre lexique de base).

\section{B. Le contexte social entourant l'échange linguistique}

Bien que plus difficiles à démontrer, les facteurs linguistiques liés au contexte social pourraient également intervenir dans une cause de libelle. En effet, des questions ayant trait aux différents niveaux de langue caractérisant les formes litigieuses pourraient être prises en considération par les parties impliquées. En d'autres mots, il est toujours possible de s'interroger sur la congruence existant entre le niveau de langue rattaché à un terme ou une expression figurant dans les propos diffamatoires prêtés à l'accusé et la situation sociale pendant laquelle s'est déroulé cet échange linguistique. Ainsi des insultes proférées en ces termes:

[3] bouché

épais

correspondent habituellement à une situation sociale dite «informelle», là où un degré minimal de surveillance de son propre parler se retrouve. Ainsi, donc, si de tels propos sont associés à une conjoncture linguistique ne se prêtant que très peu à leur production (par exemple une situation de grande formalité telle une entrevue pour un emploi, une conférence, etc.), il est permis de jeter un certain doute sur leur présence dans un tel contexte.

De la même manière, des mots généralement associés à une conversation très formelle tels:

[4] détrousseur

aigrefin

malandrin

corniaud 
pourraient difficilement apparaitre dans le cadre d'une conversation familière, surtout si celle-ci se déroule entre deux Québécois. Il devient donc possible, ici également, de contester l'apparition d'un mot inusité si le contexte entourant l'échange linguistique ne s'y prête pas.

\section{L'origine géographique du sujet}

En ce qui a trait aux facteurs d'ordre géographique, mentionnons qu'il est également possible qu'un accusé se voie prêter des propos non compatibles avec le parler caractérisant la région dont il provient. Ainsi le Montréalais qui se verrait attribuer des paroles libelleuses telles:

[5] chêti(f) ${ }^{7}$

bois-sans-soif ${ }^{8}$

Tu fais simple ${ }^{9}$ !

pourrait également être victime de témoins se parjurant. Si l'on fait abstraction d'influences très fortes, il est en effet peu probable qu'un individu adopte gratuitement le lexique d'une région qui serait autre que la sienne et qu'il n'aurait jamais habitée. Dans ce cas également, il serait approprié de fouiller les origines géographiques du ou des témoins l'incriminant dans cette affaire. Dans le cas où ces origines coïncideraient avec la région d'appartenance de l'expression ou des expressions impliquées dans la poursuite, il est raisonnable de croire qu'une défense deviendrait envisageable ici.

\section{L'âge du sujet}

Voyons maintenant comment un paramètre d'ordre chronologique pourrait exercer un rôle quelconque dans la défense ou la poursuite d'un prévenu. Prenons par exemple le cas d'un individu plutôt âgé qui en accuserait un plus jeune de l'avoir traité publiquement de voleur en ces termes:

[6] Vous n'êtes qu'un maudit larron!

Cette fois encore, une cause sans très grande défense en apparence pourrait ici, par le biais d'une étude diachronique du vocabulaire impliqué, devenir contestable devant les tribunaux. En effet, le terme sur lequel repose le libelle, soit «larron», est reconnu par la plupart des dictionnaires comme faisant partie

7. Usage caractéristique de la Mauricie et signifiant «individu désagréable, déplaisant, voire mechant».

8. Usage caractéristique de la Belgique et signifiant «ivrogne».

9. Usage caracteristique de la region du Saguenay-Lac Saint-Jean et désignant un individu đénué d'intelligence. 
d'un vocabulaire désuet, vieilli, peu employé de nos jours. Or il devient à ce moment plausible qu'une défense s'organise autour du fait que notre jeune accusé n'ait pu être enclin à l'utiliser dans son discours courant et que ce mot vieillot soit davantage issu de l'esprit du requérant dans cette cause, lui-même plus âgé et plus susceptible de conserver d'anciennes normes.

Le doute en rapport avec l'auteur possible d'une production linguistique pourra également se présenter dans le cadre d'un procès pour menaces de mort. Encore là, il sera approprié de se demander si le vocabulaire appartenant à la formulation de la menace de mort proprement dite est susceptible d'appartenir au lexique véritable de l'accusé. Comme c'était le cas pour le libelle, nous croyons que des causes d'ordre social, chronologique, géographique et stylistique (ou contextuel) peuvent apparaître ici.

Afin d'illustrer les facteurs social et stylistique, mentionnons que, sans être systématique, l'utilisation de plusieurs termes faisant fréquemment partie de la formulation de menaces de mort n'est pas laissée au hasard et dépend du statut social de son usager ou des circonstances entourant lesdites menaces de mort. Par exemple, les mots suivants :

[7] descendre zigouiller liquider avoir la peau de

qui sont tous ni plus ni moins synonymes du verbe «tuer» sont généralement associés, selon la plupart des dictionnaires que nous avons consultés, à un sujet populaire ou à une situation familière. Or il n'est pas exclu que l'on puisse remettre en doute la présence de telles formulations dans un contexte qui ne s'y prêterait guère. De la même manière, des verbes à portée sémantique similaire mais à connotation sociale différente tels:

[8] occire faucher

trucider

pourfendre

apparaîtraient difficilement dans la bouche d'un sujet peu scolarisé et dans le cadre d'une situation de grande intimité. Une certaine vigilance de l'avocat serait alors requise, surtout si le témoin incriminant l'accusé semble lui-même posséder une grande richesse lexicale (ou un lexique étendu et varié).

Mentionnons comme exemple de facteur chronologique le cas où un sujet âgé serait accusé d'avoir menacé de mort son beau-frère de la façon suivante:

[9] Le type de mort qui t'attend va être super et branché! 
D est évident qu'ici, les termes «super» et «branché» appartiennent à une génération de locuteurs qui se recrutent parmi les plus jeunes, ce qui rendrait contestable l'accusation dont serait victime cet individu âgé.

\subsubsection{Et la preuve...}

Le fait que n'importe qui ne puisse dire n'importe quoi dans n'importe quelle circonstance étant établi, il convient maintenant de se demander s'il est possible de prouver qu'un prévenu ne peut avoir dit telle ou telle phrase. Sans aller jusqu'à avancer que nous pourrions prouver que telle personne n'a pas dit telle chose, nous sommes toutefois en mesure de dire que nous pourrions semer un doute dans l'esprit du juge et du jury quant à la vraisemblance de l'accusation. Cela pourrait être fait de la façon suivante:

a) Le linguiste recueille la plus grande quantité possible de documents sonores provenant de l'accusé. Il existe toujours la possibilité que son témoignage devant la cour soit enregistré. Par ailleurs, l'on pourrait également rencontrer l'accusé avant son procès et le soumettre à différents tests en vue d'établir queis termes exacts celui-ci utiliserait dans tel ou tel contexte. Des questions du type «Quels mots emploieriez-vous pour dire que...?» ou «Quel est le synonyme de...?» ou encore «Quel est le contraire de...?» permettraient à l'expert d'avoir une excellente idée de la façon dont s'exprime généralement l'accusé. De plus, il est toujours possible de lui donner une composition française sur un sujet précis, mais cette stratégie comporte au moins deux désavantages:

1) L'usage de la langue écrite constituant en soi une situation très souvent formelle pour le sujet, il est possible que le vocabulaire y apparaissant soit légèrement différent de celui qu'il aurait utilisé au cours d'une conversation familière. Un contre-expert linguiste travaillant pour l'autre partie aura vite fait de soulever cet argument. Cela signifie qu'un individu qui, dans le cadre d'une composition française, utilise par exemple le mot «téléviseur» peut aisément employer à l'oral le mot «télévision» ou l'abréviation «T.V.».

2) Il est également très difficile de "diriger» un sujet vers un concept précis à l'intérieur d'une composition. Si par exemple l'expert cherche à découvrir comment un accusé qualifierait l'endroit où il habite (logement, logis, appartement, piaule, etc.), il lui faudra espérer qu'un peu de chance l'accompagne - à moins d'être très directif face au prévenu - s'il veut espérer retrouver l'un de ces éléments à l'intérieur de sa production écrite.

La solution idéale (mais non toujours possible) serait donc de posséder un échantillon très appréciable de productions sonores provenant de l'accusé et ayant été recueillies dans un contexte comparable à celui qui accompagnait la phrase litigieuse. 
b) Une fois ces pièces entre les mains de l'expert, il devrait lui être relativement facile d'attirer l'attention de la cour sur le peu de probabilités entourant le fait qu'un accusé puisse avoir usé de tel mot lors d'une dispute ou dans le cadre d'un libelle. $\mathrm{Si}$, par exemple, tous les documents sonores dont dispose le linguiste l'amènent à la conclusion que le prévenu emploie toujours le terme «logement» pour caractériser sa demeure, il pourrait alors affaiblir une preuve comportant la phrase:

[10] Sors de mon logis ou je te tue!

Il en serait de même dans l'éventualité où les documents sonores démontreraient l'emploi exclusif du mot «éliminer» plutôt que «tuer» dans le langage du prévenu.

\subsection{LA CONNOTATION SOCIALE ET LE MILIEU JURIDIQUE}

L'une des principales constituantes de la signification du mot a trait à la «connotation» ou contenu émotionnel du terme impliqué. Rey (1970) définit ainsi ce concept:

Élément de la signification d'une forme qui n'est pas commun à tous les communicants et peut varier selon les contextes (conçu comme affectif $v s$ intellectuel, individuel ou appartenant à un groupe restreint vs social, évocateur $v s$ descriptif, etc.) (p. 284).

Pour sa part, Gschwind-Holtzer (1981) mentionne que :

(...) la signification (est) composée d'une partie stable, générale, commune à tous les locuteurs, à laquelle s'ajoutent des sursignifications, des valeurs supplémentaires affectives, culturelles, idéologiques différenciées selon les sujets (...) (p. 13).

Ce concept linguistique et stylistique est normalement maîtrisé d'une manière presque automatique chez l'ensemble des sujets, dans le cadre de leurs échanges quotidiens. Cette situation s'explique par le fait que tout locuteur natif d'une langue connaît généralement l'effet créé par l'utilisation d'une forme ou d'une autre dans tel ou tel contexte. Malgré tout, il peut arriver qu' «une "simple" expression comme "mère de famille" qui fait gronder plus d'une féministe» comme le souligne Hagège (1985), ou qu'un énoncé tel «c'est un socialiste» soient connotés différemment selon le public devant lequel ils sont produits.

Le point sur lequel nous aimerions attirer l'attention du lecteur a trait à l'importance que devrait accorder le juriste à ce côté «affectif», «émotif» du langage, ce côté «imagination et émotions» qui s'oppose peut-être à l'intelligence (Lyons, 1970). Cela signifie donc qu'en plus de se soucier du contenu 
(ou signifié) du message qu'il doit véhiculer, l'avocat (et dans certains cas le témoin "préparé» par l'avocat) aurait tout intérêt à manifester une certaine vigilance eu égard aux formulations employées devant la cour, au choix des mots, aux formes syntaxiques prononcées tout au long des interrogatoires, des plaidoiries, des témoignages.

Cet ajustement devrait être fait en fonction du contexte précis dans lequel évolue le juriste. Nous incluons dans la notion de "contexte» des éléments aussi variés que le profil sociolinguistique du témoin interrogé par l'avocat, celui du jury ainsi que le rôle joué par l'avocat dans le cadre du procès (poursuite ou défense). Voyons brièvement comment ces trois éléments contextuels devraient influencer la valeur connotative des formes utilisées devant la cour.

a) Le profil sociolinguistique du témoin interrogé. L'avocat qui procède à l'interrogatoire d'un témoin dans le but de lui «délier la langue» devrait toujours garder à l'esprit que plus son langage s'apparentera à celui du témoin, plus ce dernier sera à l'aise, en climat de confiance, donc susceptible d'afficher une très grande volubilité. À preuve cet avocat de la Commission d'enquête sur le crime organisé qui, il y a quelques années, questionnant un témoin qui parlait de «boîtes de cartron» (sic), se mit, lui aussi, à utiliser le terme «cartron» jusqu'à la fin de son interrogatoire, et ce avec des résultats étonnants.

Par ailleurs, le juriste questionnant un témoin pouvant apparaître sympathique ou vulnérable auprès du magistrat (ou, à plus forte raison, auprès du jury) ne devrait jamais perdre de vue que le pouvoir dominateur que lui confère le fait d'être celui qui interroge pourra aussi bien lui nuire que l'aider face à ceux qui jugeront. Ainsi que le mentionne Gschwind-Holtzer (1981):

L'acte d'interroger (...) se classe parmi les actes d'autorité dans la mesure où il oblige l'autre à entrer, quelle que soit sa motivation personnelle, dans l'univers discursif. Interroger, c'est exercer une pression sur l'auditeur en le poussant dans les voies de la parole. L'acte d'autorité qu'est la question s'affirme avec le plus de force dans le cadre de l'interrogatoire officiel ou privé où questionner devient synonyme de soupçonner, accuser, est donc attaque contre l'autre (p. 38).

C'est pourquoi il nous apparaît impératif pour le juriste de manipuler ce pouvoir avec soin, avec prudence, sans en abuser de façon apparemment préjudiciable pour le témoin. À l'instar de Gschwind-Holtzer, nous croyons qu'il est possible que:

(...) la dépendance statutaire se marque (...) par des actes d'autorité pratiqués sous une forme atténuée propre à effacer le trait de "pouvoir». La demande de faire quelque chose s'exprimera alors non par l'impératif, 
trop direct, mais à l'aide de formules et de circonlocutions énoncées avec une intonation particulière (ibid).

b) Le profil sociolinguistique du jury. De la même façon que les publicistes optent généralement pour un langage s'apparentant à celui de la clientèle cible, l'avocat qui interroge et qui plaide devant un jury devrait toujours se soucier de ce que la connotation sociale se rattachant à son langage corresponde le plus possible à celui que prônent la majorité des jurés lui faisant face (ou le «leader» de ce jury, le cas échéant).

Ainsi, dans le cas où l'avocat est confronté à un jury composé majoritairement de gens peu scolarisés, il lui serait sans doute plus utile de s'exprimer dans un langage relativement simple, clair, limitant les mots «savants» ou les tournures trop recherchées. Si, au contraire, la plupart des jurés ont une scolarité appréciable, le juriste aura tout intérêt à tendre vers une langue plus châtiée, plus standard. Les remarques formulées ci-dessus s'appliquent également aux témoins appelés par l'avocat.

c) Le rôle tenu par l'avocat dans le cadre du procès. Nous avons déjà mentionné le fait que la langue présente peu de vrais synonymes. Selon Ullmann, seuls les mots pouvant se substituer l'un à l'autre dans tous les contextes sans le moindre changement de sens cognitif ou «connotatif» peuvent être appelés synonymes. Et c'est précisément sur ce dernier point que nous voudrions insister. Il arrivera fréquemment que deux ou plusieurs mots entretiennent entre eux un rapport de quasi-synonymie; toutefois, c'est au niveau de la connotation ou de l'effet créé chez l'interlocuteur que se manifestera la plus grande différence entre eux. C'est pourquoi, selon l'effet que voudra créer l'avocat sur le jury ou, en d'autres mots, selon le rôle qu'il assumera dans le procès (poursuite ou défense), il est de première importance qu'il use des différents «synonymes» en usant de prudence, voire de stratégie.

Par exemple, dans le cadre d'un procès, l'emploi de l'une ou l'autre des formes suivantes par les avocats de la Défense ou de la Couronne, créera, pour qualifier le même meurtre, un impact fort différent:

[11] à la suite de l'incident du $1^{\text {er }}$ décembre (connotation très faible);

[12] à la suite des événements du $1^{\mathrm{er}}$ décembre (terme neutre);

[13] à la suite du massacre du $1^{\text {er }}$ décembre (connotation très forte).

L'avocat assumant la défense d'un prévenu aurait tout intérêt à opter pour [12], l'énoncé [11] minimisant beaucoup trop l'importance du geste (ce qui risquerait de heurter le jury et de le «rebeller» contre le procureur) et l'énoncé [13] comportant une connotation pouvant visiblement nuire à son client. Quant à l'avocat de la poursuite, il aurait sans doute avantage à adopter la formulation 
[13], pour les mêmes raisons. Ces remarques s'appliquent également aux témoins appelés par les différents avocats.

\section{CONCLUSION}

La présence du témoin-expert linguiste en cour de justice relève d'un phénomène tout à fait récent, du moins au Canada. Et cette situation n'est certainement pas étrangère au fait que le passage d'un tel témoin devant le magistrat fait quelquefois l'objet, pendant la période d'approbation surtout, de questions ayant trait à la pertinence d'un tel témoignage. Généralement, le linguiste aura tôt fait de dissiper toutes les incertitudes relatives à son implication en faisant valoir le fait que de même que la géographie est la science qui étudie les phénomènes physiques situés à la surface du globe terrestre, la linguistique n'est rien d'autre que l'étude scientifique du langage. Et qui dit étude «scientifique» dit «d'une valeur universelle, caractérisée par un objet et une méthode déterminés, et fondée sur des relations objectives vérifiables ${ }^{10}$ ».

La langue est en effet un objet d'étude qui, souventes fois, provoque chez celui qui manifeste pour lui de l'intérêt une réaction empreinte d'émotivité, de subjectivité, faisant alors obstacle à une analyse objective. C'est alors que surgissent les campagnes de bon parler, les jugements de valeur, les sentiments d'autodéfense contre l' «envahisseur», etc. La linguistique s'inscrit tout à fait à l'encontre de cette approche, cherchant plutôt à faire ressortir les «universaux » du langage, les constantes émanant d'études rigoureusement menées, dénuées de toute opinion à caractère arbitraire, et faisant preuve d'une précision et d'une minutie ne pouvant être mises en doute.

Cette recherche s'effectuera, en milieu judiciaire, au moyen d'une approche phonétique, où l'étude instrumentale des sons de la parole pourra nous permettre de déterminer clairement la nature d'un phonème ou même, dans certains cas et avec les précautions qui s'imposent, la paternité ou la nonpaternité de propos litigieux. C'est également par le biais de l'analyse lexicologique, celle-là même qui se préoccupe de la «structure» des mots, de leurs relations sémantiques, que pourra s'opérer une analyse rigoureuse du vocabulaire apparaissant dans les contrats comme les testaments, les menaces de mort aussi bien que les libelles. C'est aussi à travers la lunette syntaxique, témoin des liens unissant les mots en phrases, que pourra se poursuivre l'étude du «discours juridique».

Finalement, une expertise à caractère sociolinguistique, reposant sur les liens unissant langage et société, pourra nous permettre d'établir les rapports

10. Le Petit Robert (1987). 
existant entre le profil socio-économique d'un prévenu (défini à partir de critères tels l'âge, le sexe, la scolarité, le milieu d'origine, etc.) et le type de production linguistique auquel l'on serait en droit de s'attendre. Dans le cas où survient une incompatibilité, confirmée par une vérification du discours, parlé ou écrit, antérieur et ultérieur au délit, une possible influence extérieure, lors de la déclaration statutaire, ou un faux témoignage, s'il s'agissait d'une accusation de libelle ou de menaces de mort, devient alors plausible. Par ailleurs, la connaissance et la maîtrise des diverses connotations sociales caractérisant différents mots, différentes tournures utilisés devant la cour pourront sans aucun doute constituer un atout majeur pour l'avocat désireux d'inciter un témoin à s'exprimer ou de convaincre juge et jury de la justesse de ses propos.

La sociolinguistique ne constitue pas une nouvelle arme pour l'avocat; celle-ci était déjà entre ses mains. Nous croyons toutefois que cette science est en mesure de lui fournir l'occasion de saisir toute la dimension structurelle de la langue, tout ce côté prévisible, non aléatoire se rattachant à cet ensemble de symboles indispensables à la complexité de la communication humaine. Et ce n'est qu'une fois ce postulat connu et reconnu par le milieu juridique que ce dernier pourra véritablement exploiter les innombrables ressources se rapportant à l'analyse sociolinguistique en cour de justice.

\section{BIBLIOGRAPHIE}

BASSNET-McGUIRE, S. (1980), Translation Studies, Londres, Methuen.

BÉDARD, E., MAURAIS, J. (1983), La Norme linguistique, Québec, Gouvernement du Québec, Conseil de la langue française.

BERNSTEIN, Basil (1975), Langage et classes sociales, Paris, Éditions de Minuit.

BLOOMFIELD, Lénard (1970), Le Langage, Paris, Payot.

BOURDIEU, P., BOLTANSKI, L. (1975), «Le fétichisme de la langue», Actes de recherches en sciences sociales, vol. 4, pp. 2-33.

COSTERMANS, Jean (1980), Psychologie du langage, Bruxelles, Pierre Mardaga editeur.

DUFLOT-FAVORI, C. (1988), Le Psychologue expert en justice, Paris, Presses universitaires de France.

FISHMAN, J. (1968), Readings in the Sociology of Language, La Haye, Mouton.

GARMADI, Juliette (1981), La Sociolinguistique, Paris, Presses universitaires de France.

GSCHWIND-HOLTZER, Gisèle (1981), Analyse sociolinguistique de la communication et didactique, Paris, Hatier.

HAGEGE, Claude (1985), L'Homme de paroles, Paris, Fayard.

HERTZLER, J. (1965), A Sociology of Language, New York, Random House. 
LABOV, William (1976), Sociolinguistique, Paris, Éditions de Minuit.

LABOV, William (1978), Le Parler ordinaire, Paris, Éditions de Minuit.

LAROQUE, Pierre (1972), Les Classes sociales, Paris, Presses universitaires de France.

LECLERC, Jacques (1986), Langue et société, Laval, Mondia Éditeurs.

LYONS, John (1970), Linguistique générale. Introduction a la linguistique théorique, Paris, Larousse.

MARCELLESI, J.-B., GARDIN, B. (1974), Intraduction d la sociolinguistique, Mont SaintAignan, Cahiers de linguistique sociale.

MOUNIN, Georges (1972), Clefs pour la sémantique, Paris, Seghers.

REY, Alain (1970), La Lexicologie, Paris, Klincksieck.

RICHAUDEAU, François (1981), Linguistique pragmatique, Paris, Retz.

TOUSIGNANT, Claude (1987), La Variation sociolinguistique, modèle québécois et méthode d'analyse, Sillery, Presses de l'Université du Québec.

ULLMANN, Stephen (1957), The Principles of Semantics, Glasgow, Jackson et Oxford, Basil Blackwell, vol. II. 